\title{
CK0403, a 9-aminoacridine, is a potent anti-cancer agent in human breast cancer cells
}

\author{
YUAN-WAN SUN ${ }^{1}$, KUEN-YUAN CHEN ${ }^{2}$, CHUL-HOON KWON $^{3}$ and KUN-MING CHEN ${ }^{1}$ \\ ${ }^{1}$ Department of Biochemistry and Molecular Biology, College of Medicine, Pennsylvania State University, Hershey, \\ PA 17033, USA; ${ }^{2}$ Department of Surgery, National Taiwan University Hospital, Taipei 8862, Taiwan, R.O.C.; \\ ${ }^{3}$ Department of Pharmaceutical Sciences, College of Pharmacy and Allied Health Professions, \\ St. John's University, New York, NY 11439, USA
}

Received November 11, 2014; Accepted October 22, 2015

DOI: $10.3892 / \mathrm{mmr} .2015 .4604$

\begin{abstract}
Acridin-9-ylamino)phenylthio]phenyl $\}$ (3-hydroxypropyl)amino)propan-1-ol (CK0403) is a sulfur-containing 9-anilinoacridine analogue of amsacrine and was found to be more potent than its analogue 2-(\{4-[4-(acridin9-ylamino)phenylthio]phenyl\} (2-hyd roxyethyl)a mino) ethan-1-ol (CK0402) and amsacrine in the inhibition of the topoisomerase II-catalyzed decatenation reaction. A previous study by our group reported that CK0402 was effective against numerous breast cancer cell lines, and the combination of CK0402 with herceptin enhanced its activity in HER2(+) SKBR-3 cells. In order to identify novel chemotherapeutic agents with enhanced potency, the present study explored the potential of CK0403 in the treatment of breast cancer. First, the growth inhibitory activity of CK0403 in the breast cancer cell lines MCF-7, MDA-MB-231, BT474 and SKBR-3, as well as in the non-cancerous MCF-10A cell line, was examined using a sulforhodamine B assay. The results showed that CK0403 exerted more potent growth inhibitory activity than CK0402 in all of the breast cancer cell lines except MCF-7. SKBR-3 and MDA-MB-231 were the most sensitive cell lines tested, and the combination of CK0403 with herceptin in HER2(+) SKBR-3 cells enhanced the growth inhibitory activity of CK0403. Analysis of cell cycle alterations induced by CK0403 in SKBR-3 cells revealed that, similarly to CK0402, CK0403 induced $\mathrm{G}_{2} / \mathrm{M}$-phase arrest with a decreased $\mathrm{S}$ - and $\mathrm{G}_{0} / \mathrm{G}_{1}$-phase ratio. In addition, it was shown that $\mathrm{CK} 0403$ induced apoptosis more effectively than CK0402 in SKBR-3 cells. Further analysis of autophagy protein 5 (Atg5) indicated that CK0403 induced more cleaved Atg5 than CK0402 and other chemotherapeutic agents tested. Of note, although still relatively potent, CK0403 exhibited
\end{abstract}

Correspondence to: Dr Kun-Ming Chen, Department of Biochemistry and Molecular Biology, College of Medicine, Pennsylvania State University, Hershey, PA 17033, USA

E-mail:kzc3@psu.edu

Key words: topoisomerase II inhibitor, CK0402, CK0403, breast cancer, apoptosis, autophagy, 9-aminoacridines reduced growth inhibitory activity under hypoxic conditions, which can induce autophagy. Collectively, the present results supported that CK0403 is highly potent and more effective than CK0402 against estrogen receptor-negative and HER2-overexpressing breast cancer cell lines, suggesting its future application for chemotherapy in breast cancer.

\section{Introduction}

Breast cancer is the most common type of cancer diagnosed in American women and is the second most common cause of cancer-associated mortality. In 2014, 232,670 new breast cancer cases are expected to be diagnosed, and 40,430 breast cancer mortalities are expected in the USA (1). In the treatment of breast cancer, anthracyclines (e.g. doxorubicin and daunorubicin) are among the most active anti-neoplastic agents in use (2-5). Although highly effective in chemotherapy, the mechanism of action of these compounds is not specific and several mechanisms have been demonstrated, including inhibition of topoisomerase I/II and production of reactive oxygen species (ROS). In addition, clinical use of anthracyclines is often hampered by its cumulative dose-limiting cardiotoxicity, as well as the development of drug resistance by tumor cells $(6,7)$. Following the success of doxorubicin, a tremendous amount of effort has been made in the search of effective alternatives; however, only few of them are clinically approved and none of them are clearly superior to doxorubicin $(8,9)$. Therefore, the development of effective therapeutic strategies, including alternatives to anthracyclines and/or their combination with other effective agents, remains an important task in breast cancer treatment.

The anti-cancer agent amsacrine (m-AMSA; Fig. 1) is a topoisomerase II inhibitor and is structurally a 9-anilinoacridine analogue. 9-Anilinoacridines have displayed an advantage over other topoisomerase II inhibitors in that they were not affected by transport-mediated multidrug resistance (MDR). In addition, atypical MDR can be overcome by structure modification at its topoisomerase II-binding domain (10). In an attempt to develop improved chemotherapeutic agents, 9-anilinoacridine has been subjected to various structural modifications on the acridine backbone as well as on the aniline ring. It is generally thought that the mode of action of 


\section{Chart 1}

(CH)

1. $\mathrm{n}=2, \mathrm{CK} 0402$

2. $\mathrm{n}=3, \mathrm{CK} 0403$<smiles>COc1cc(NS(C)(=O)=O)ccc1Nc1c2ccccc2nc2ccccc12</smiles>

3. Amsacrine

Figure 1. Chemical structures of (1) CK0402, (2) CK0403 and (3) amsacrine.

this type of compound is through DNA intercalations facilitated by the tricyclic acridine moiety; additional interactions between the aniline ring of the compound with other molecules can lead to the inhibition of topoisomerase II $(11,12)$.

A previous study by our group reported the synthesis and preliminary anti-cancer evaluation of a series of sulfur-containing 9-anilinoacridine derivatives, including 2-(\{4-[4-(acridin-9-ylamino) phenylthio]phenyl\} (2-hydroxyethyl)amino)ethan-1-ol (CK0402) and 3-(\{4-[4-(acridin-9-ylamino) phenylthio]phenyl\}(3-hydroxypropyl)amino)propan-1-ol (CK0403) (Fig. 1) (13). Within a library of sulfur-containing 9-anilinoacridines, CK0403 and CK0402 were the most cytotoxic agents against Chinese hamster lung transformed V79 cells (13). Similarly to amsacrine and anthracyclines, CK0403 inhibited a topoisomerase II $\alpha$-catalyzed decatenation reaction, and it was $\sim 10$ times more effective than amsacrine and its analogue CK0402 according to study by our group (14). A further study by our group showed that CK0402 was effective against numerous breast cancer cell lines; in addition, the combination of CK0402 with herceptin enhanced its activity in HER2(+) SKBR-3 cells (15). Reduced production of ROS was noted in CK0403-treated fibroblast cells compared with that in cells treated with CK0402; furthermore, the cell proliferation inhibitory activity of CK0403 was enhanced when combined with NU1025, a potent and specific poly(adenosine diphosphate-ribose polymerase 1 inhibitor (14). In addition, a clonogenic assay demonstrated that CK0403 was more potent than CK0402 against hepatocellular carcinoma HepG2 cells; however, in breast cancer MCF-7 cells, the activity of CK0403 and CK0402 was comparable (14).

To examine whether CK0403 is more active than CK0402 against breast cancer cell lines other than MCF7, the present study first evaluated the cytotoxic effect of CK0403 on a panel of established human breast cell lines with varying levels of estrogen receptor (ER), progesterone receptor (PR) and HER2/neu, the most common clinically used biomarkers for breast cancer treatment. The human breast cell lines used included MCF-7 [ER(+), HER2(-) and PR(+)], BT-474 [ER(+), HER2 overexpressing and PR(+)], MDA-MB-231 [ER(-), HER2(-), PR(-)] and SKBR-3 [ER(-), HER2 overexpressing, $\mathrm{PR}(-)]$, and the non-cancerous MCF-10A cell line [ER(-), HER2(-), PR(-)]. The cytotoxicity of CK0403 was subsequently evaluated on the most sensitive cell line, SKBR-3, under hypoxic conditions or with the combination of herceptin. The potential underlying mechanisms of the cytotoxic effects of CK0403 were also explored using cell cycle analysis, quantification of the apoptotic rate and assessment of autophagy-associated signaling.

\section{Materials and methods}

Chemicals and reagents. CK0403 was prepared and purified as described previously (13). Herceptin was kindly provided by Genentech Inc. (San Francisco, CA, USA). Dimethyl sulfoxide (DMSO, cell culture grade), sulforhodamine B and propidium iodide were purchased from Sigma-Aldrich Chemical Co. (St. Louis, MO, USA). Dulbecco's modified Eagle's medium (DMEM)/F12, Iscove's modified Dulbecco's medium(IMDM), horse serum, penicillin/streptomycin, phosphate-buffered saline (PBS), RNase A and trypsin-EDTA were purchased from Gibco-BRL (Invitrogen Life Technologies, Inc. Carlsbad, CA, USA). Fetal bovine serum (FBS) was purchased from Gemini Bio-Products (Woodland, CA, USA).

Cell culture and treatment. All of the cell lines were obtained from the American Type Culture Collection (Manassas, VA, USA) and grown in $75-\mathrm{cm}^{2}$ cell culture flasks at $37^{\circ} \mathrm{C}$ in a humidified atmosphere of $5 \% \mathrm{CO}_{2}$ in air. MCF-7 and MDA-MB-231 cells were maintained in IMDM/F12 (1:1 mixture) medium; SKBR-3 and BT-474 cells were maintained in DMEM/F12 (1:1 mixture). All the media were supplemented with penicillin/streptomycin $(50 \mathrm{mg} / \mathrm{ml})$ and $10 \% \mathrm{FBS}$. MCF-10A cells were maintained in DMEM/F-12 supplemented with $5 \%$ horse serum, insulin $(10 \mathrm{mg} / \mathrm{ml})$, hydrocortisone (500 ng/ml), epidermal growth factor $(20 \mathrm{ng} / \mathrm{ml})$, cholera toxin $(100 \mathrm{ng} / \mathrm{ml})$ and penicillin/streptomycin $(50 \mathrm{mg} / \mathrm{ml})$. For hypoxia treatment, cells were incubated in $1 \% \mathrm{O}_{2} / 5 \% \mathrm{CO}_{2} / 94 \%$ $\mathrm{N}_{2}$ overnight prior to drug treatment. Hypoxic conditions were generated using a PROOX sensor (model C21; BioSpherix, Hanover, NH, USA) using $\mathrm{N}_{2}$ influx. Drug treatment involved continuous incubation with the compounds for six days. For all cell culture experiments, cells were seeded at least $24 \mathrm{~h}$ prior to treatment.

Cell proliferation assay. Cells ( 3,000-5,000/well) were seeded and allowed to grow in a 96-well plate for at least $24 \mathrm{~h}$ prior to treatment. DMSO was used as a vehicle to dissolve CK0403 with a final DMSO concentration $\leq 0.1 \%(\mathrm{v} / \mathrm{v})$ in the medium. Herceptin was added $3 \mathrm{~h}$ prior to the addition 
Table I. LC $_{50}$ values of CK0403 in a panel of breast cancer cell lines and the normal breast cell line MCF10A.

\begin{tabular}{lcc}
\hline Cell line & $\mathrm{LC}_{50}(\mu \mathrm{M})^{\mathrm{a}}$ & Immunoprofile \\
\hline MCF-7 & $1.06 \pm 0.35$ & $\mathrm{ER}^{+}, \mathrm{HER}^{-}, \mathrm{PR}^{+}$ \\
MDA-MB-231 & $0.09 \pm 0.02$ & $\mathrm{ER}^{-}, \mathrm{HER} 2^{-}, \mathrm{PR}^{-}$ \\
BT-474 & $0.94 \pm 0.53$ & $\mathrm{ER}^{+}, \mathrm{HER}^{+}, \mathrm{PR}^{+}$ \\
SKBR-3 & & $\mathrm{ER}^{-}, \mathrm{HER}^{+}, \mathrm{PR}^{-}$ \\
$\begin{array}{l}\text { Normoxia } \\
\text { Hypoxia }\end{array}$ & $0.07 \pm 0.05$ & \\
MCF10A & $0.12 \pm 0.03$ & \\
& $4.25 \pm 0.93$ & $\mathrm{ER}^{-}, \mathrm{HER} 2^{-}, \mathrm{PR}$
\end{tabular}

ancubation was performed for six days. Growth inhibition was determined using the sulforhodamine B assay. Values are expressed as the mean \pm standard deviation from three independent experiments. $\mathrm{LC}_{50}$, concentration leading to a $50 \%$ reduction in cell survival. ER, estrogen receptor; PR, progesterone receptor.

of CK0403 when cells were treated with the combination of CK0403 and herceptin. At the end of the incubation, cultures were fixed with $50 \mu 150 \%$ cold trichloroacetic acid and incubated at $4^{\circ} \mathrm{C}$ for $1 \mathrm{~h}$. The plates were washed five times with water and then air-dried. The fixed cells were stained for $30 \mathrm{~min}$ with $100 \mu 10.4 \%$ sulforhodamine B solution in $1 \%$ acetic acid. The plates were then washed five times with $1 \%$ acetic acid to remove any unbound dye. The bound dye was dissolved with $10 \mathrm{mM}$ Tris buffer and the absorbance of the resulting solution was measured at $570 \mathrm{~nm}$ to quantify the number of surviving cells. All of the treatments were performed at least three times in triplicate. The drug concentration that produced a $50 \%$ reduction $\left(\mathrm{LC}_{50}\right.$ ) in cell survival was determined using median-effect plots.

Apoptotic cell death detection. To quantitatively determine the apoptotic cell death induced by CK0403, CK0402 and amsacrine (Sigma-Aldrich Chemical Co.), a cell death detection ELISA kit (Roche Diagnostics, Indianapolis, IN, USA) was used and enrichment factor was reported, according to the manufacturer's instructions. Briefly, following treatment of cells with the indicated drugs at 0.3125 or $0.525 \mu \mathrm{M}$ for $72 \mathrm{~h}$, the cells were lysed and incubated for $30 \mathrm{~min}$ at room temperature; aliquots of $20 \mu \mathrm{l}$ supernatant were then transferred into the wells of a streptavidin-coated microtiter plate. To each well, $80 \mu \mathrm{l}$ immunoreagent was added. After incubation at room temperature for $2 \mathrm{~h}$, the solution was decanted, and each well was rinsed three times with incubation buffer. Color development was performed by adding $100 \mu \mathrm{l}$ 2,2'-azino-bis(3-ethylbenzothiazoline-6-sulfonic acid (ABTS) solution and absorbency was measured at $405 \mathrm{~nm}$ in a microtiter plate reader (Spectramax Plus 384; Molecular Devices, Sunnyvale, CA, USA) against ABTS solution as a blank.

Flow cytometric cell cycle analysis. SKBR-3 cells growing in the exponential phase were treated with the indicated concentrations of CK0403 or DMSO as previously described (15). At the end of the incubation, cells were harvested, fixed in ice-cold $70 \%$ ethanol and stored at $-20^{\circ} \mathrm{C}$. The fixed cells were washed with phosphate-buffered saline, treated with RNase A (3 units $/ \mathrm{ml}$ ) at $37^{\circ} \mathrm{C}$ for $30 \mathrm{~min}$ and stained with propidium iodide $(50 \mathrm{mg} / \mathrm{ml})$ for $5 \mathrm{~min}$. DNA content for 250,000 cells per analysis was monitored using a Becton-Dickinson FACScan flow cytometer (BD Biosciences, Franklin Lakes, NJ, USA) and Modfit software (LT version 2.0; Verity Software House, Topsham, ME) was used for analysis of the cell cycle distribution.

Western blot analysis. SKBR-3 cells were treated with CK0403, CK0402, amsacrine, doxorubicin or camptothecin (Sigma-Aldrich Chemical Co.) at $1 \mu \mathrm{M}$ for $24 \mathrm{~h}$. Cells were harvested by scraping and washed with PBS. Cellular proteins were isolated with cell lysis buffer purchased from Cell Signaling Technology, Inc (Beverly, MA, USA). Equal amounts of protein $(40 \mu \mathrm{g})$ were boiled for $5 \mathrm{~min}$, separated by $10 \%$ SDS-PAGE at $100 \mathrm{~V}$ for $110 \mathrm{~min}$ and then electro-transferred onto polyvinylidene difluoride membranes. Antibody against Atg5 was purchased from Novus (Littleton, CO, USA; rabbit polyclonal; cat. no. NB110-53818; $1: 3,000)$. $\beta$-Actin antibody (as a reference standard) was purchased from Sigma-Aldrich (mouse monoclonal; cat. no. A5316; 1:5,000). Primary antibody incubations were performed at $4^{\circ} \mathrm{C}$ overnight. Following extensive washing, specific bands were detected using immobilon western chemiluminescent substrate (Millipore, Billerica, MA, USA). Secondary anti-rabbit (cat. no. 7076) or anti-mouse (cat. no. 7074) immunoglobulin $\mathrm{G}$ conjugated to horse radish peroxidase were purchased from Cell Signaling Technology, Inc.

Statistical analysis. The statistical comparisons between certain pairs of measurements were performed using Student's t-test. Statistical analyses were performed using Microsoft Excel 2007. The data are expressed as the mean \pm standard deviation.

\section{Results}

CK0403 inhibits the growth of human breast cancer cells. The human breast cancer cell lines MCF-7, MDA-MB-231, BT-474 and SKBR-3 were selected to evaluate the growth inhibitory activity of CK0403. Based on a previous study by our group on CK0402, the cells were incubated with CK0403 for six days (15). In addition, the growth inhibitory effect of CK0403 on the non-cancerous human breast cell line MCF-10A was examined. As shown in Table I, MCF-10A was the cell line with the lowest sensitivity to CK0403 treatment. Among the breast cancer cell lines tested, the magnitude of the growth inhibitory effects of CK0403 was in the following order: SKBR3 $\geq$ MDA-MB-231>BT-474 $\geq$ MCF-7.

A previous study by ourgroup reported thatCK0402induced apoptosis and autophagy in SKBR-3 ells (15). Autophagy is induced by a variety of metabolic stresses, including hypoxia and oxidative stress. To elucidate the role of autophagy in the cell death induced by CK0403, the growth inhibitory activity of CK0403 in SKBR-3 cells was examined under normoxic and hypoxic conditions in parallel. The results showed that CK0403 was less potent under hypoxic conditions as compared with normoxia $\left(\mathrm{LC}_{50}=0.12 \mu \mathrm{M}\right.$ vs. $0.07 \mu \mathrm{M}$, respectively). 
Table II. Potentiation of the growth inhibition activity of CK0403 by herceptin in SKBR-3 human breast cancer cells ${ }^{\mathrm{a}}$.

\begin{tabular}{lccc}
\hline & \multicolumn{3}{c}{ Percentage of cell survival (\%) } \\
\cline { 2 - 4 } Concentration of CK0403 $(\mu \mathrm{M})$ & CK0403 & Herceptin $^{\mathrm{b}}$ & CK0403 + Herceptin \\
\hline 0.03125 & 50.3 & 75.9 & 45.3 \\
0.125 & 39.5 & 91.6 & 26.1 \\
1 & 19.8 & 69.2 & 4.3 \\
\hline
\end{tabular}

${ }^{a}$ Cells were continuously incubated with these agents for six days. ${ }^{b}$ The dose of herceptin was $1 / 20$ of the respective concentration of CK0403.

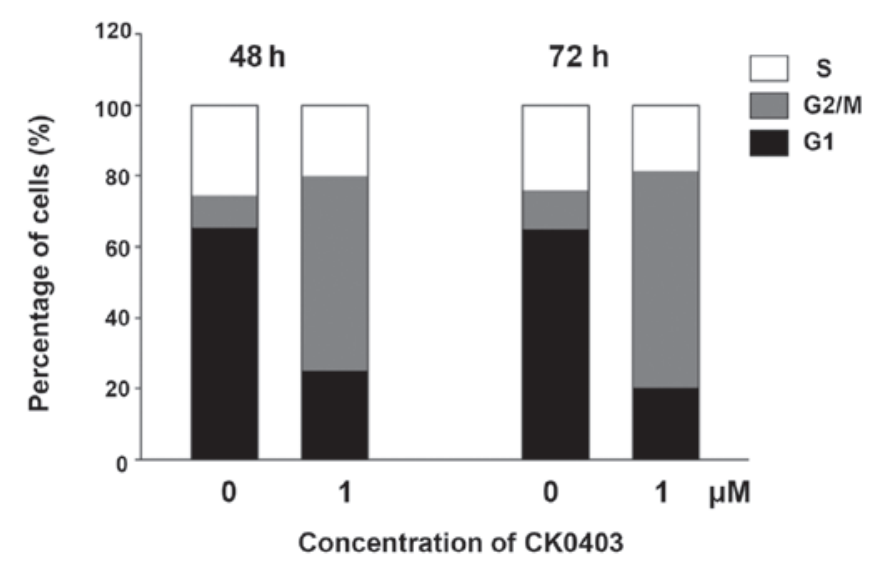

Figure 2. Effects of CK0403 on the cell cycle progression of SKBR-3 breast cancer cells. Cells were stained with propidium iodide and analyzed by flow cytometry. The results are representative data from three independent experiments.

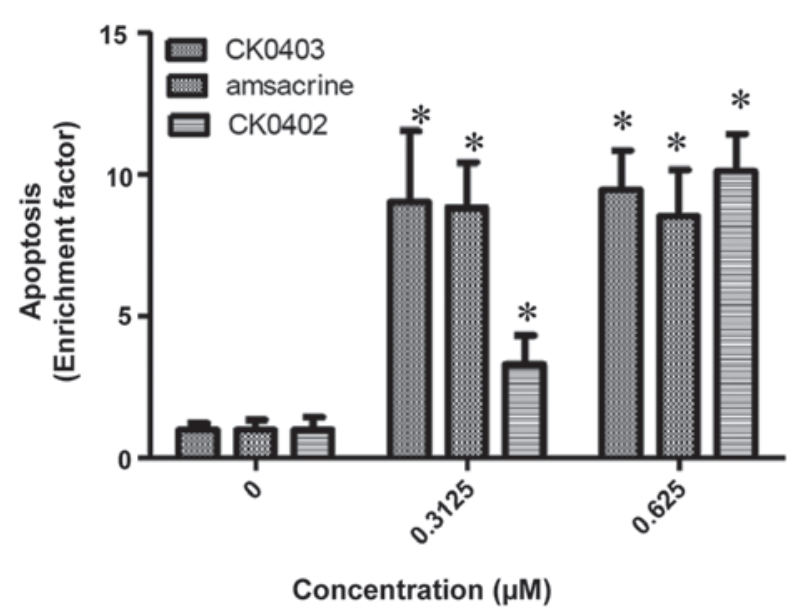

Figure 3. Induction of apoptosis by CK0402, CK0403 and amsacrine in SKBR-3 breast cancer cells analyzed by ELISA. SKBR-3 cells were incubated with the respective compounds at 0.3125 or $0.525 \mu \mathrm{M}$ for $72 \mathrm{~h}$. The data are expressed as the mean \pm standard deviation $\left({ }^{*} \mathrm{P}<0.05\right.$, compared with the control).

CK0403 induces $G_{2} / M$ phase arrest in SKBR-3 cells. In order to examine the cell cycle distribution of SKBR-3 cells, cells were treated with CK0403 at $1 \mu \mathrm{M}$ for 48 or $72 \mathrm{~h}$, stained with propidium iodide and subjected to flow cytometric analysis (Fig. 2). The results showed that CK0403 time-dependently induced $\mathrm{G}_{2} / \mathrm{M}$ arrest with a reduced $\mathrm{G}_{1}$ - and $\mathrm{S}$-phase population

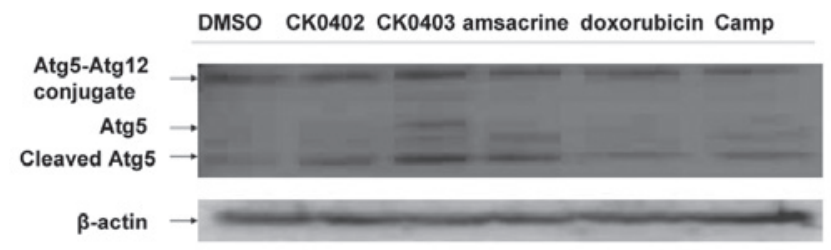

Figure 4. Induction of Atg5 protein expression by CK0403 in SKBR-3 breast cancer cells. A representative western blot is shown. The concentration used for all the tested agents other than DMSO was $1 \mu \mathrm{M}$. DMSO, dimethyl sulfoxide (vehicle control); Atg, autophagy protein; Camp, camptothecin.

in SKBR-3 cells. These results are similar to those observed for CK0402; however, it was noted that CK0403 induced a lower increase in the $\mathrm{G}_{2} / \mathrm{M}$-phase population as compared to CK0402.

CK0403 induces apoptosis in SKBR-3 breast cancer cells. The cell growth inhibition resulting from CK0403 treatment was further characterized by evaluating the apoptotic response induced by CK0403 and compared with that of CK0402 and amsacrine in SKBR-3 cells using an ELISA kit (Fig. 3) (15). As it was previously found that CK0402 did not induce apoptosis effectively within $48 \mathrm{~h}$ of treatment, apoptosis was analyzed at $72 \mathrm{~h}$. The results showed that CK0403 induced apoptotic effects in SKBR-3 cells at doses of 0.3125 and $0.625 \mu \mathrm{M}$, resulting in a 9.02 \pm 2.51 - and 9.47 \pm 1.39 -fold enhancement of apoptosis, respectively. The results of the present study showed that at the dose of $0.3125 \mu \mathrm{M}, \mathrm{CK} 0403$ induced apoptosis in SKBR-3 cells more effectively than its analogue CK0402, but equally effective as amsacrine. At the dose of $0.625 \mu \mathrm{M}$, all three compounds were equally effective at inducing apoptosis in SKBR-3 cells.

CK0403 induces the autophagic pathway in SKBR-3 cells. Atg5 cleavage provokes apoptotic cell death, and it represents a molecular link between autophagy and apoptosis (16). As shown in Fig. 4, the present study demonstrated that treating SKBR3 cells with CK0403 at the dose of $1 \mu \mathrm{M}$ led to the induction of cleaved Atg5 protein expression. Furthermore, it was shown that at the same dose, CK0403 induced higher levels of cleaved Atg5 protein than other anti-cancer agents tested, including CK0402, amsacrine, doxorubicin and camptothecin.

Herceptin potentiates the CK0403-induced growth inhibition of SKBR-3 cells. As shown in Fig. 1, among the cell 
lines tested in the present study, the HER2-overexpressing SKBR-3 cell line was most sensitive to CK0403 treatment. Since SKBR-3 cells overexpress the HER2 receptor, SKBR-3 cells were selected to evaluate the combined effects of CK0403 and herceptin. At the tested doses, the growth inhibitory effects of CK0403 on SKBR-3 cells were found to be potentiated by the herceptin added at $1 / 20$ of the concentration of CK0403 (Table II).

\section{Discussion}

Structurally, CK0402, CK0403 and amsacrine are 9-aminoacridine analogues. Amsacrine was the first clinically successful synthetic DNA-intercalating agent (8). Amsacrine and its analogs contain a DNA-binding domain (acridine moiety) and a topoisomerase II-binding domain (aniline moiety). Amsacrine itself, distinct from anthracyclines, is not affected by transport-mediated MDR; in addition, its topoisomerase II-binding domain can be modified to overcome the observed atypical MDR (10). Thus, structural modifications of amsacrine analogs may provide effective second-line therapeutics for tumors that have developed resistance to doxorubicin and etoposide. Certain amsacrine analogues have been found to be potent against the human breast carcinoma T-47D cell line and showed activity in phase II clinical studies on breast cancer $(17,18)$. In spite of these successes, continuous efforts are made to develop novel 9-aminoacridine analogues with enhanced activity against breast cancer in pre-clinical studies $(13,14,19-21)$.

A previous study by our group showed that CK0402 possesses activity against a broad spectrum of cancer types, including a panel of established human breast cancer cell lines (15). CK0403 is an analogue of CK0402 and was found to inhibit the topoisomerase-II $\alpha$ catalyzed decatenation reaction $\sim 10$ times more effectively than amsacrine and its CK0402 analogue (14). The present study, determined that CK0403 is more potent than its CK0402 analogue against several breast cancer cell lines, particularly to the HER2overexpressing SKBR-3 cell line and the triple-negative MDA-MB-231 cell line. Of note, the non-cancerous MCF10A cell line was the least sensitive cell line among the cell lines tested, indicating that CK0403 may selectively kill malignant cells over non-malignant cells. Based on the growth-inhibitory effects induced by CK0403 on the panel of cell lines examined in the present study, CK0403 appears to be more effective against malignant ER(-) cells than against ER(+) cells.

The tumor microenvironment has a crucial role in the chemoresistance of tumor cells. A hypoxic environment in solid tumors is common, which results in increased anaerobic glycolysis, new blood vessel formation, genetic instability and a decreased responsiveness to radio- as well as chemotherapy $(22,23)$. Therefore, tumor hypoxia represents a challenge for chemotherapeutic agents. Autophagy is induced by a variety of metabolic stresses, including hypoxia and oxidative stress. Increasing evidence has shown that autophagy contributes to the resistance to anti-neoplastic agents, including topoisomerase II inhibitors $(24,25)$. A previous study by our group showed that autophagy as well as apoptosis can be induced by CK0402 in SKBR-3 cells (15); however, the role of autophagy in CK0402-induced cytotoxic effects has remained elusive. The results of the present study indicated that the growth inhibitory activity of CK0403 was reduced, although not distinctly, under hypoxic conditions. Whether autophagy contributes to the cellular resistance to cell death induced by CK0403 will be the subject of further study. The $\mathrm{LC}_{50}$ value was in the nanomolar range under hypoxic as well as normoxic conditions, demonstrating that CK0403 effectively inhibited the growth of SKBR-3 cells under either condition.

The present study further demonstrated that CK0403 induced the cleavage of Atg 5 in SKBR-3 cells more effectively than CK0402, amsacrine, doxorubicin and camptothecin at the doses used. The process of autophagosome formation requires the covalent conjugation of Atg12 and Atg5 in a ubiquitylation-like process (26). However, the non-conjugated forms of Atg12 and Atg5 are involved in the induction of apoptosis; in apoptotic cells, Atg5 is cleaved by calpains and then translocates to the mitochondria, resulting in the release of cytochrome $\mathrm{C}$ by interacting with B-cell lymphoma 2 family proteins $(16,26)$.

The present and a previous study by our group showed that CK0402 and CK0403 induced $\mathrm{G}_{2} / \mathrm{M}$ arrest in breast cancer cells, which is a common cellular response to treatment with DNA-damaging agents (15). In addition, the sensitivity of various breast cancer cell lines to CK0403 was consistent with that to CK0402. A previous study by our group determined that apoptosis was the type of cell death induced by CK0402 in breast cancer cells. In a similar fashion, CK0403 effectively induced apoptosis in SKBR-3 cells, as demonstrated in the present study. Based on the above results and the structural similarity between the two compounds, it is concluded that CK0403 inhibited breast cancer cell growth through similar mechanisms to those of CK0402, but with higher potency. Collectively, our the results of the present study supported that CK0403 is superior to $\mathrm{CK} 0402$ as a potential treatment option for ER-negative and HER2-overexpressing breast cancers; furthermore, the growth inhibitory effects on HER2-overexpressing breast cancer cells were potentiated by the addition of herceptin.

\section{Acknowledgements}

The present study was supported by a grant from the Susan G. Komen Breast Cancer Foundation (no.BCTR0707876) and the Tobacco settlement fund from the Pennsylvania Department of Health.

\section{References}

1. Cancer Facts \& Figures. 2014. American Cancer Society, Atlanta, 2014.

2. Gianni L and Valagussa P: Anthracyclines and early breast cancer: The end of an era? J Clin Oncol 27: 1155-1157, 2009.

3. O'Shaughnessy J: Liposomal anthracyclines for breast cancer: Overview. Oncologist 8 (Suppl 2): S1-S2, 2003.

4. Qian BJ, Yan F, Li N, Liu QL, Lin YH, Liu CM, Luo YP, Guo F and Li HZ: MTDH/AEG-1-based DNA vaccine suppresses lung metastasis and enhances chemosensitivity to doxorubicin in breast cancer. Cancer Immunol Immunother 60: 883-893, 2011.

5. Weiss RB: The anthracyclines: Will we ever find a better doxorubicin? Semin Oncol 19: 670-686, 1992.

6. Carvalho FS, Burgeiro A, Garcia R, Moreno AJ, Carvalho RA and Oliveira PJ: Doxorubicin-induced cardiotoxicity: From bioenergetic failure and cell death to cardiomyopathy. Med Res Rev 34: 106-135, 2014.

7. Ng R, Better N and Green MD: Anticancer agents and cardiotoxicity. Semin Oncol 33: 2-14, 2006. 
8. Denny WA: DNA-intercalating ligands as anti-cancer drugs: Prospects for future design. Anticancer Drug Des 4: 241-263, 1989.

9. Minotti G, Menna P, Salvatorelli E, Cairo G and Gianni L: Anthracyclines: Molecular advances and pharmacologic developments in antitumor activity and cardiotoxicity. Pharmacol Rev 56: 185-229, 2004.

10. Baguley BC, Holdaway KM and Fray LM: Design of DNA intercalators to overcome topoisomerase II-mediated multidrug resistance. J Natl Cancer Inst 82: 398-402, 1990.

11. Denny WA: Acridine derivatives as chemotherapeutic agents. Curr Med Chem 9: 1655-1665, 2002.

12. Nelson EM, Tewey KM and Liu LF: Mechanism of antitumor drug action: Poisoning of mammalian DNA topoisomerase II on DNA by 4'-(9-acridinylamino)-methanesulfon-m-anisidide. Proc Natl Acad Sci USA 81: 1361-1365, 1984.

13. Chen KM, Sun YW, Tang YW, Sun ZY and Kwon CH: Synthesis and antitumor activity of sulfur-containing 9-anilinoacridines. Mol Pharm 2: 118-128, 2005.

14. Park SK, Kang $\mathrm{H}$ and Kwon $\mathrm{CH}$ : Caspase-dependent cell death mediates potent cytotoxicity of sulfide derivatives of 9-anilinoacridine. Anticancer Drugs 19: 381-389, 2008

15. Sun YW, Niu TK, Yang JM, Kwon CH, Chen KY and Chen KM: Potentiation of growth inhibition activity of 2-(\{4-[4-(acridin-9-ylamino)phenylthio]phenyl\}(2-hydroxyethyl) amino) ethan-1-ol (CK0402) by Herceptin in SKBR-3 human breast cancer cells. Exp Ther Med 1: 513-518, 2010.

16. Yousefi S, Perozzo R, Schmid I, Ziemiecki A, Schaffner T, Scapozza L, Brunner T and Simon HU: Calpain-mediated cleavage of Atg5 switches autophagy to apoptosis. Nat Cell Biol 8: 1124-1132, 2006.

17. Baguley BC, Denny WA, Atwell GJ, Finlay GJ, Rewcastle GW, Twigden SJ and Wilson WR: Synthesis, antitumor activity and DNA binding properties of a new derivative of amsacrine, N-5-dimethyl-9-[(2-methoxy-4-methylsulfonylamino) phenylamino]-4-acridinecarboxamide. Cancer Res 44: 3245-3251, 1984.

18. Fyfe D, Price C, Langley RE, Pagonis C, Houghton J, Osborne L, Woll PJ, Gardner C, Baguley BC and Carmichael J; Cancer Research Campaing Phase I/II Trials Committee: A phase I trial of amsalog (CI-921) administered by intravenous infusion using a 5-day schedule. Cancer Chemother Pharmacol 47: 333-337, 2001.
19. Bacherikov VA, Chou TC, Dong HJ, Zhang X, Chen $\mathrm{CH}$, Lin YW, Tsai TJ, Lee RZ, Liu LF and Su TL: Potent antitumor 9-anilinoacridines bearing an alkylating $\mathrm{N}$-mustard residue on the anilino ring: Synthesis and biological activity. Bioorg Med Chem 13: 3993-4006, 2005.

20. Kapuriya N, Kapuriya K, Zhang X, Chou TC, Kakadiya R, Wu YT, Tsai TH, Chen YT, Lee TC, Shah A, et al: Synthesis and biological activity of stable and potent antitumor agents, aniline nitrogen mustards linked to 9-anilinoacridines via a urea linkage. Bioorg Med Chem 16: 5413-5423, 2008.

21. Su TL, Lin YW, Chou TC, Zhang X, Bacherikov VA, Chen $\mathrm{CH}$, Liu LF and Tsai TJ: Potent antitumor 9-anilinoacridines and acridines bearing an alkylating $\mathrm{N}$-mustard residue on the acridine chromophore: Synthesis and biological activity. J Med Chem 49: 3710-3718, 2006.

22. Chun YS, Adusumilli PS and Fong Y: Employing tumor hypoxia for oncolytic therapy in breast cancer. J Mammary Gland Biol Neoplasia 10: 311-318, 2005.

23. Adamski JK, Estlin EJ and Makin GW: The cellular adaptations to hypoxia as novel therapeutic targets in childhood cancer. Cancer Treat Rev 34: 231-246, 2008.

24. Sui X, Chen R, Wang Z, Huang Z, Kong N, Zhang M, Han W, Lou F, Yang J, Zhang Q, et al: Autophagy and chemotherapy resistance: A promising therapeutic target for cancer treatment. Cell Death Dis 4: e838, 2013.

25. Tamura N, Hirano K, Kishino K, Hashimoto K, Amano O, Shimada J and Sakagami H: Analysis of type of cell death induced by topoisomerase inhibitor SN-38 in human oral squamous cell carcinoma cell lines. Anticancer Res 32: 4823-4832, 2012.

26. Rubinstein AD and Kimchi A: Life in the balance-a mechanistic view of the crosstalk between autophagy and apoptosis. J Cell Sci 125: 5259-5268, 2012. 